\title{
Synthesis and Characterization of Liquid Crystal Elastomer
}

\author{
Rita A. Gharde1, Santosh A. Mani1,2, Suman Lal'3,4, Samriti Khosla3 , S. K. Tripathi ${ }^{4}$ \\ ${ }^{1}$ Department of Physics, University of Mumbai, Mumbai, India \\ ${ }^{2} \mathrm{~K}$. J. Somaiya College of Engineering, Vidyavihar (E), Mumbai, India \\ ${ }^{3}$ Department of Physics, J.C.D.A.V College, Dasuya, Punjab, India \\ ${ }^{4}$ Department of Advanced Study, Panjabi University, Chandigarh, India \\ Email: gharde.rita@gmail.com, samphy2013@gmail.com
}

Received 3 April 2015; accepted 12 June 2015; published 15 June 2015

Copyright (C) 2015 by authors and Scientific Research Publishing Inc.

This work is licensed under the Creative Commons Attribution International License (CC BY).

http://creativecommons.org/licenses/by/4.0/

(c) (i) Open Access

\begin{abstract}
The thermal and mechanical properties of Liquid Crystal Elastomers (LCEs) were characterized using various techniques for understanding of their physical behavior. The material used for investigation was synthesized by us, using Finklemann procedure, with proper cross linking density in nematic phase. The material is found to have unique coupling between anisotropicorder of liquid crystal component and elasticity of polymer network. The chemical structures were confirmed by Fourier Transform Infrared (FTIR) Spectroscopy and Scanning Electron Microscopy (SEM). Fabry Perot Scattering Studies (FPSS), Thermo gravimetric Analysis (TGA) and Differential Scanning Calorimatory (DSC) were used to study thermal properties. The mechanical properties were studied using force sensor. Our investigation shows that this synthesized Liquid Crystal Elastomer has ability of spontaneous change as a function of temperature and mechanical force, which shows it as a unique class of soft material.
\end{abstract}

\section{Keywords}

Liquid Crystal Elastomers (LCEs), Fourier Transform Infrared (FTIR) Spectroscopy, Scanning Electron Microscopy (SEM), Fabry Perot Scattering Studies (FPSS), Thermo gravimetric Analysis (TGA), Differential Scanning Calorimetry (DSC)

\section{Introduction}

Liquid Crystal Elastomers (LCEs) are new class of soft materials which combine the elastic properties of polymers and orientational properties of Liquid Crystals. They consist of the cross linked polymer chain networks and the crystalline ordering of Liquid Crystal. Thus it introduces a coupling between the orientation of the me- 
sogens and macroscopic elastic deformations of the network [1] [2].

LCE brings together three important properties: orientational order in amorphous soft materials, responsive molecular shape and quenched topological constraints, which helps to create many new physical phenomenon and have attracted significant interest from scientific community due to their fascinating thermal, mechanical, optical and electrical properties. The concept of LCE was put forward by the P.G Gennes, where as, the first LCE was synthesized by Finkelmann. LCEs are divided into nematic, smectic and others depending on the LC phases exhibited by the systems. In nematic phase, the polymer chains elongate, while in the isotropic phase, they recover a random coil conformation, which is driven by their entropy [3]-[5].

In recent years the emphasis is being given on the synthesis and characterization of LCE. The studies of various physical stimuli, LCEs with thermo-responsive, photo-responsive and electro-responsive functions have been developed and LCEs as artificial muscles, micropumps and microvalves for microfluidic devices and optomechanical shutters have been actively explored [6]-[10]. Finkelmann et al. measured visco-elastic, opto-elastic and their behavior in electric field; Tajbakhsh et al. studied uniaxial monodomain Nematic elastomers. Terentjev et al. studied a wide range of photo sensitive materials; Dannio et al. studied thermo-tropic and elastic properties. In one way or other, many new physical properties of these soft materials have been discovered [11]-[16].

In this work, we synthesized LCE using Finklemann procedure and the characterizations were performed by optical, thermal and mechanical techniques to understand the structure-property relation. These investigations confirm synthesis of LCE and the spontaneous effect on relative movement of their cross-linked end points results in the elastic response of the material which is very important for optimizing properties and achieving real applications.

\section{Experimental}

The synthesis of these elastomers were made Finklemann procedure by preparing partially crosslinked films in a centrifuge, highly swollen in toluene ( $2-3 \mathrm{ml}$ per $1 \mathrm{~g}$ of material), reacting for 25 - 35 minutes before evaporating the solvent. A careful study of reaction kinetics ensured that approximately $50 \%$ of crosslinks were established in the first stage of this preparation. The orientation is then fixed by the subsequent second-stage reaction, when the remaining crosslinks are fully established. The structure of polymer, monomer and cross linking agent is shown in Figure 1.

\section{Results and Discussion}

\subsection{FTIR Spectroscopy Analysis}

The synthesis of LCE were confirmed by FTIR spectra obtained from Perkin Elemer spectrometer in the spectral region ranging from 4000 to $400 \mathrm{~cm}^{-1}$. The FTIR spectra of synthesized LCE is shown in Figure 2. The bands in between 3445 and $2926 \mathrm{~cm}^{-1}$ is due to benzene C-H bond, bands at 2926 and $2857 \mathrm{~cm}^{-1}$ is due to long chain of $-\mathrm{CH}_{2}$ - group, band at $1731 \mathrm{~cm}^{-1}$ is due to $\mathrm{C}=\mathrm{O}$ group, band at $1606 \mathrm{~cm}^{-1}$ is due to $\mathrm{C}=\mathrm{C}$ group, band at 1509 $\mathrm{cm}^{-1}$ is due to benzene $(\mathrm{C}=\mathrm{C})$, band at $1465 \mathrm{~cm}^{-1}$ is $\mathrm{C}-\mathrm{H}$ deforming, $1256 \mathrm{~cm}^{-1}$ is due to $\mathrm{Si}-\mathrm{C}$ bond, $1196 \mathrm{~cm}^{-1}$ is due to C-O (sample present in the group), $1069 \mathrm{~cm}^{-1}$ is due to C-O of benzene group, $1009 \mathrm{~cm}^{-1}$ is $\mathrm{Si}-\mathrm{O}$ bonding, band at $763 \mathrm{~cm}^{-1}$ also confirms benzene in the sample. This spectrum confirms the synthesis of LCE.

\subsection{SEM Analysis}

The SEM images of the LCE at $10 \mu \mathrm{m}$ and $30 \mu \mathrm{m}$ scale for Everhart-Thornley (EHT) bias of $20 \mathrm{KV}$ is shown in Figure 3. The most prominent feature is referred as the coarse structure of $10 \mu \mathrm{m}$ scale. This is due to polymer network attached to the material. The structure of polymer, monomer and cross linking agent is also seen at 30 $\mu \mathrm{m}$ scale.

In these photographs, crosslinking are oriented axially and the anisotropic nodes lie in the different orientations. SEM investigation revealed nematic nature of LCE. The surface of LCE also shows bend and give way under pressure or strain due to contraction. The elastic deformation related effects can be observed and also confirms the fact that degree of orientation of these materials can be controlled by varying crosslinking density.

\subsection{FPSS Analysis}

The LCE was heated by an indigenous electric heater characterized using He-Ne laser of power $2 \mathrm{~mW}$. The light 


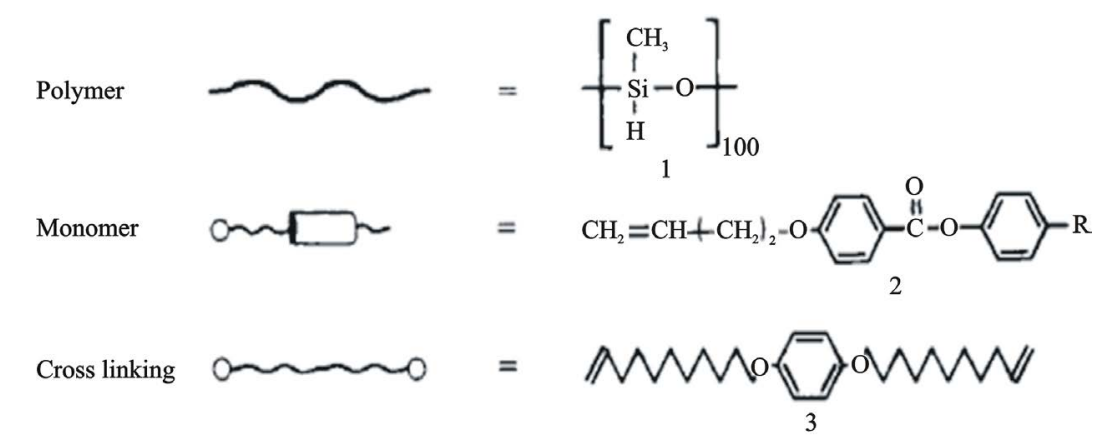

Figure 1. Synthesis of LCE.

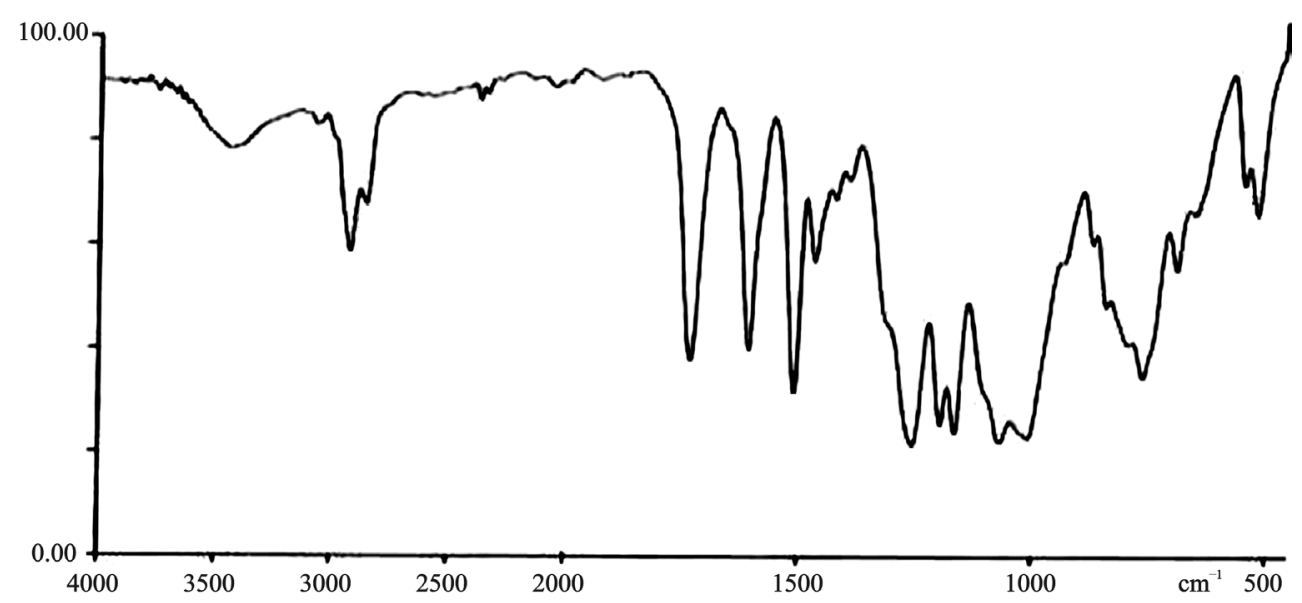

Figure 2. FTIR spectra of LCE.
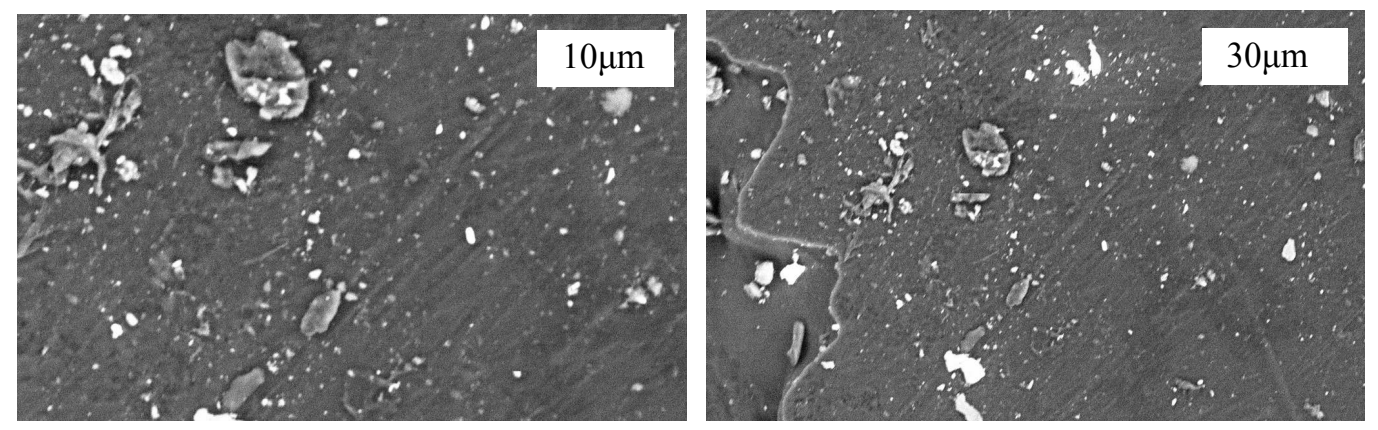

Figure 3. SEM image of LCE.

scattered by the LCE sample was allowed to fall on the Fabry-perot Etalon and the angular diameters of the rings were measured at various temperatures. The experiment was repeated for five heating/cooling cycles for consistency. The graphical mapping of Angular diameter Vs Temperature for only one cycle is shown Figure 4. (other graphs can be provided if required). The abrupt variation in diameter shows mesophase transitin temerature of sample.

\subsection{TGA Analysis}

The thermal characterization of sample is performed by Perkin Elmer TGA. The analysis was made at heating rate of $10^{\circ} \mathrm{C} / \mathrm{min}$. The weight is recorded as a function of increasing temperature from $30^{\circ} \mathrm{C}$ to $150^{\circ} \mathrm{C}$. For TGA, the rate of mass change with time is described by the Equation:

$$
\mathrm{d} W / \mathrm{d} t=A \mathrm{e}^{-E / R T} W^{n}
$$


where: $W$ is the dimensionless mass of sample subjected to degradation is time, $A$ is a pre-exponential factor, $E$ is the activation energy and $n$ is the effective reaction order. The TGA graph of LCE is shown in Figure 5. It was observed that weight remains constant up to of $60^{\circ} \mathrm{C}$ and again after $120^{\circ} \mathrm{C}$. The linear change in weight is due to spontaneous nature of the LCE.

\subsection{DSC Analysis}

DSC measures temperature differences in the heat flow path to the sample and reference material by constant rate heating. The DSC thermo graph of LCE for heating and cooling cycle is shown in Figure 6. The DSC signal can be expressed by the following Equation.

$$
\mathrm{d} Q / \mathrm{d} t=-C p \cdot(\mathrm{d} T / \mathrm{d} t)+K(T, t)
$$

where, $\mathrm{d} Q / \mathrm{d} t$ is DSC Total Heat Flow, $\mathrm{d} T / \mathrm{d} t$ is Heating rate, $C p$ is Specific Heat Capacity of sample $t$ is Time, $T$ is Temperature and $K(T, t)$ is Heat flow change with behavior.

The heat flow (in $\mathrm{mW}$ ) was recorded up to temperature of $100^{\circ} \mathrm{C}$ at a heating rate of $10^{\circ} \mathrm{C} / \mathrm{min}$. The phase transition temperature observed by DSC is in agreement with FPSS and it also confirms spontaneous nature of the LCE characterized by TGA analysis.

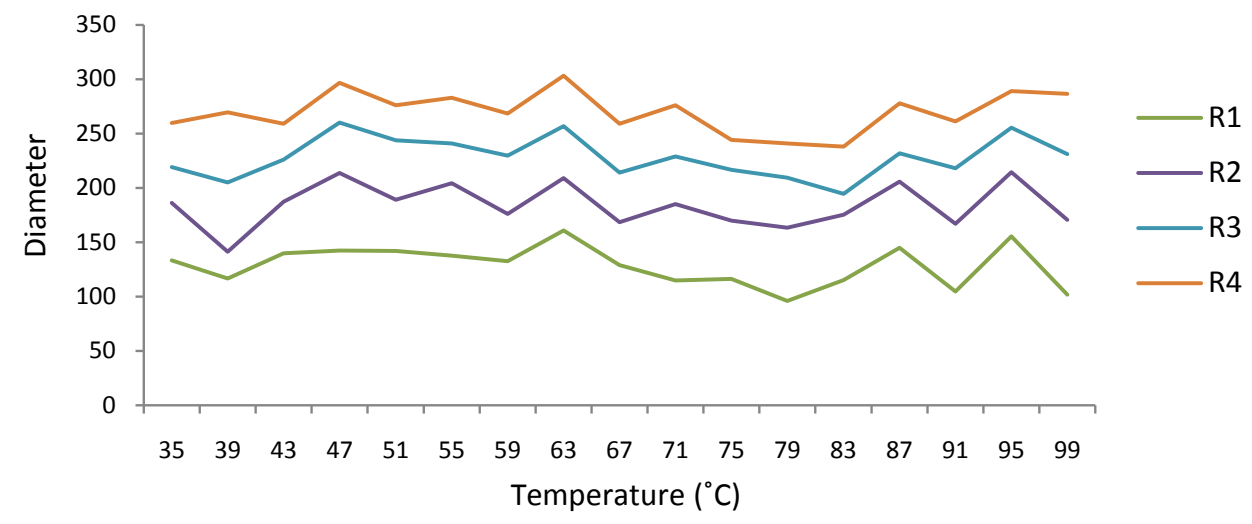

Figure 4. FPSS graph of LCE.

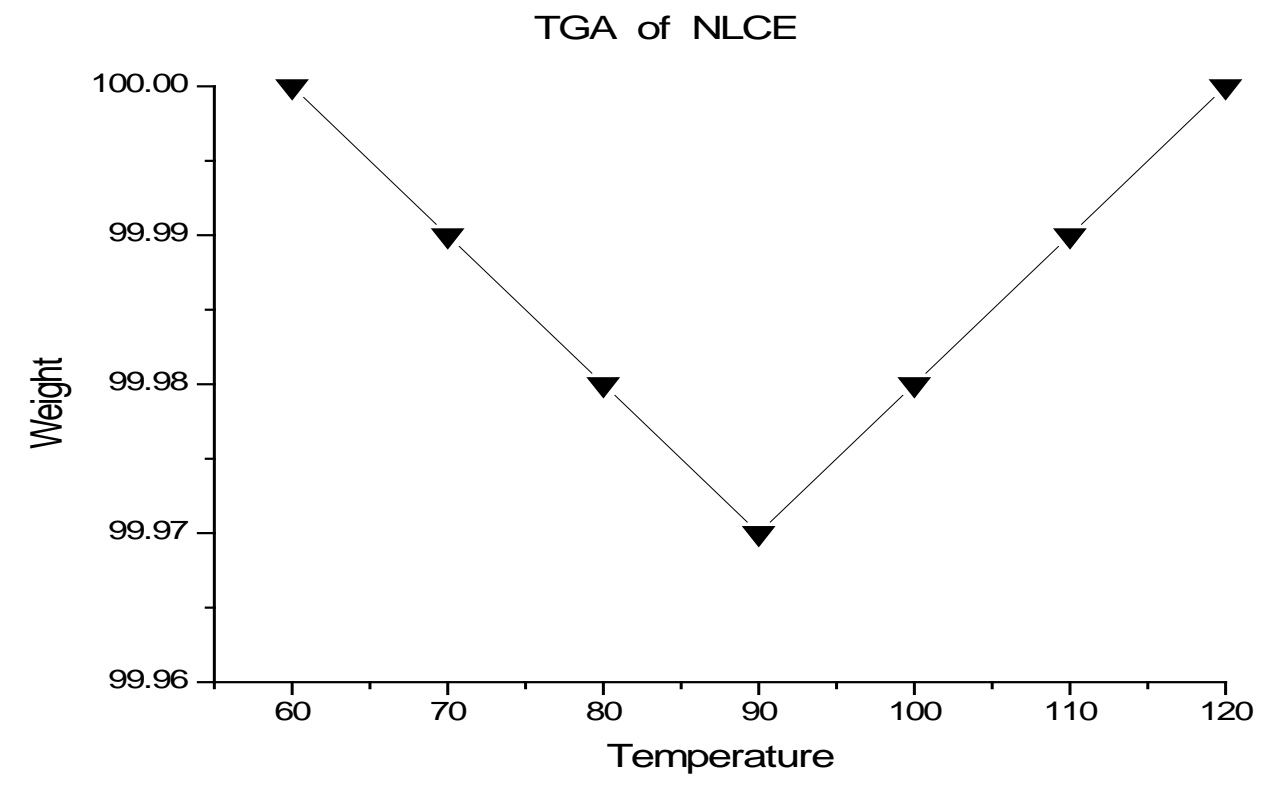

Figure 5. TGA graph of LCE. 


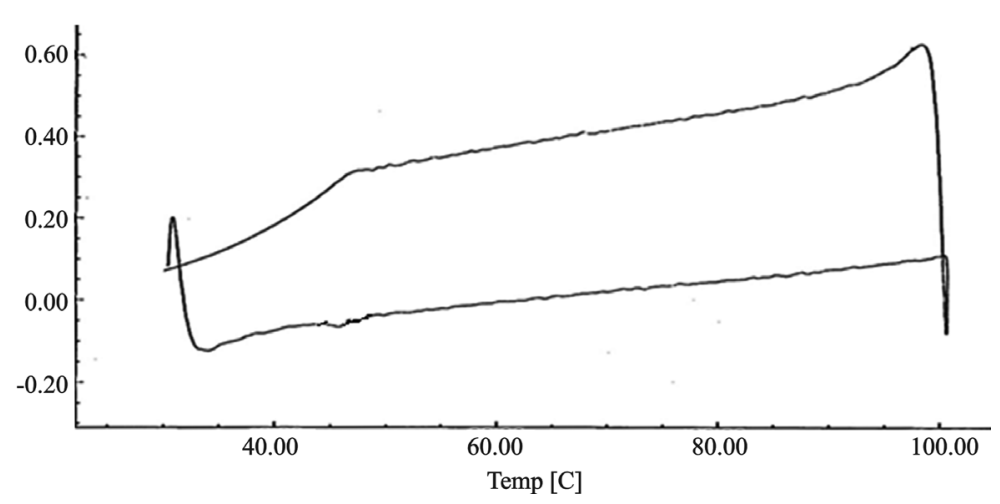

Figure 6. DSC graph of LCE.

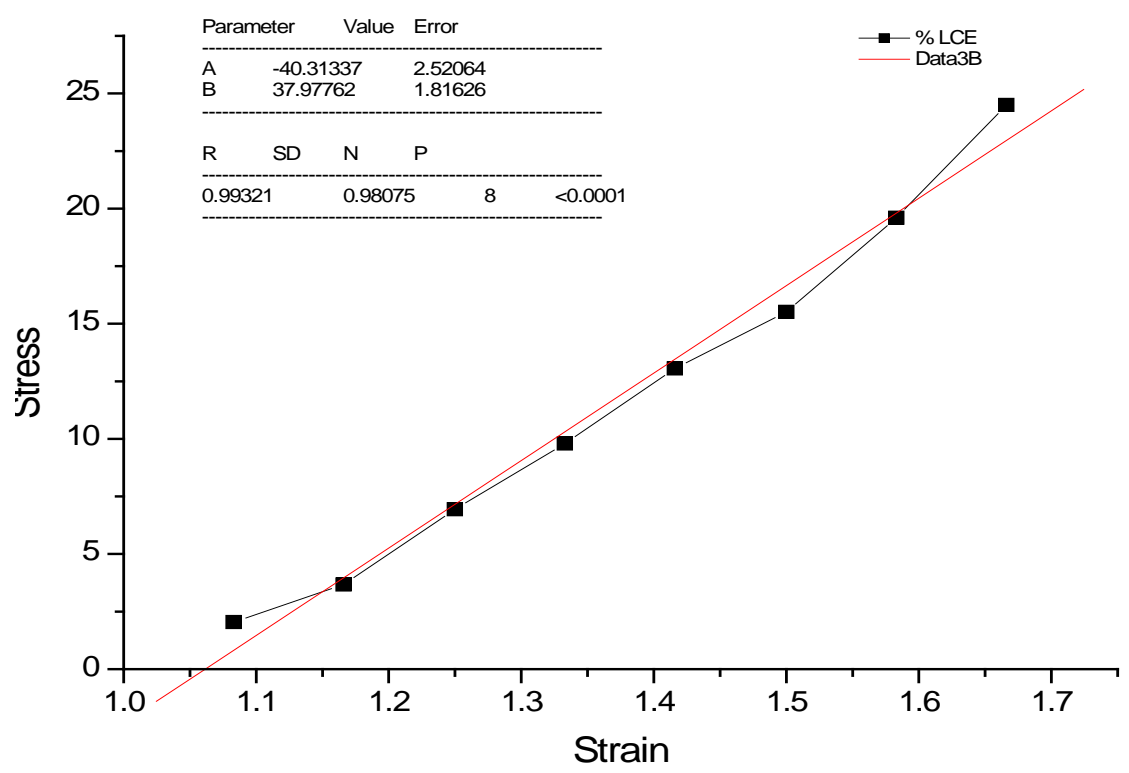

Figure 7. Stress strain-graph of LCE.

\subsection{Mechanical Studies}

When mechanical forces are applied to LCE materials, they deform in reaction to those forces. The magnitude of the deformation depends on force. The length and breadth of sample were taken as $5 \mathrm{~mm}$, thickness $1 \mathrm{~mm}$ and area $25 \mathrm{~mm}^{2}$. The stress strain-graph of LCE studied by Force sensor is shown in Figure 7. The graph shows linear relation and hence obeys Hooke's Law. The strain can be thought of as a normalized deformation. This shows elastic response of LCE.

\section{Conclusion}

The spectroscopic techniques confirm the synthesis whereas a thermal and mechanical characterization shows elastic and spontaneous nature of LCE. This is a unique property where a macroscopic shape change costs little elastic free energy. This spontaneous change of LCE leads to many applications such as thermo-mechanical actuators and artificial muscles. The control of optical birefringence of LCEs makes them suitable for optomechanical sensors and the fact that they are rather soft mechanically and still retain their shape as solids, makes them highly suitable for bifocal contact and intra-ocular lenses.

\section{Acknowledgements}

We would like to acknowledge the help and encouragement given to us by Dr. Anuradha Misra, Professor and 
Head, Department of Physics, University of Mumbai, Mumbai.

\section{References}

[1] De Gennes, P.G. (1975) The Physics of Liquid Crystals. Oxford University Press, Oxford.

[2] Zental, R. and Reckert, G. (1986) Liquid Crystalline Elastomers Based on Liquid Crystalline Side Group, Main Chain and Combined Polymers. Die Makromolekulare Chemie, 187, 1915-1926.

http://dx.doi.org/10.1002/macp.1986.021870811

[3] Terentjev, E.M. (1999) Liquid-Crystalline Elastomers. Journal of Physics: Condensed Matter, 11, 239-257. http://dx.doi.org/10.1088/0953-8984/11/24/201

[4] Legge, C.H., Davis, J. and Mitchell, G.R. (1991) Memory Effects in Liquid Crystal Elastomers. Journal de Physique, 21, 1253-1261. http://dx.doi.org/10.1051/jp2:1991131

[5] Finkelman, H. and Kupfer, J. (1991) Nematic Liquid Single Crystal Elastomers. Die Makromolekulare Chemie, Rapid Communications, 12, 717-726. http://dx.doi.org/10.1002/marc.1991.030121211

[6] Fridrikh, S.V. and Terentjev, E.M. (1999) Polydomain-Monodomain Transition in Nematic Elastomers. Physical Review E, 60, 1847. http://dx.doi.org/10.1103/PhysRevE.60.1847

[7] Tajbakhsh, A.R. and Terentjev, E.M. (2001) Spontaneous Thermal Expansion of Nematic Elastomers. The European Physical Journal E, 6, 181-188. http://dx.doi.org/10.1007/s101890170020

[8] Finkelmann, H., Koch, H.J. and Rehange, G. (1981) Investigation of Liquid Crystalline Polysiloxanes 3. Liquid Crystalline Elastomers-A New Type of Liquid Crystalline Materials. Die Makromolekulare Chemie, Rapid Communications, 2, 317-322.

[9] Bispo, M., Guillon, D., Donnio, B. and Finkelmann, H. (2008) Main-Chain Liquid Crystalline Elastomers: Monomer and Cross-Linker Molecular Control of the Thermotropic and Elastic Properties. Macromolecules, 41, 3098-3108. http://dx.doi.org/10.1021/ma7026929

[10] Hogan, P.M., Tajbakhsh, A.R. and Terentjev, E.M. (2001) UV-Manipulation of Order and Macroscopic Shape in Nematic Elastomers. Cond. Mat.

[11] Perline, R., Kornbluh, R., Pei, Q. and Joseph, J. (2000) High-Speed Electrically Actuated Elastomers with Strain Greater Than 100\%. Science, 287, 836-839.

[12] Warner, M. and Terentjev, E.M. (2003) Liquid Crystal Elastomers. Clarendon Press, Oxford.

[13] David, P.K. and Fritz, V. (2002) Biological Liquid Crystal Elastomers. Philosophical Transactions of the Royal Society $B, 357,155-163$.

[14] Dukes, D., Li, Y., Lewis, S., Benicewicz, B., Schadler, L. and Kumar, S.K. (2010) Conformational Transitions of Spherical Polymer Brushes: Synthesis, Characterization, and Theory. Macromolecules, 43, 1564-1570. http://dx.doi.org/10.1021/ma901228t

[15] Biggins, J.S., Warner, M. and Bhattacharya, K. (2012) Elasticity of Polydomain Liquid Crystal Elastomers. Journal of the Mechanics and Physics of Solids, 60, 573-590. http://dx.doi.org/10.1016/j.jmps.2012.01.008

[16] Jayaraman, A.J. (2013) Polymer Grafted Nanoparticles: Effect of Chemical and Physical Heterogeneity in Polymer Grafts on Particle Assembly and Dispersion. Journal of Polymer Science Part B: Polymer Physics, 51, 524-534. http://dx.doi.org/10.1002/polb.23260 